Results Primarily we compared thickness of subcutaneous adipose tissue in girls-carriers of different genotypes in both groups. Girls with android obesity - carriers of AA genotype, thickness of subcutaneous adipose tissue in breast was 1.1 $\pm 0.2 \mathrm{~cm}$; AG genotype - $0.94 \pm 0.2 \mathrm{~cm}$; GG-genotype - 0.93 $\pm 0.2 \mathrm{~cm}$ ( $\left.\mathrm{p}^{\mathrm{AA}-\mathrm{AG} ; \mathrm{AA}-\mathrm{GG}}=0.04\right)$. Metabolism parameters: insulin in carriers of AA genotype was 25.2 \pm 13.6 ; AG genotype $15.5 \pm 8.3$; GG genotype $-18.1 \pm 11.4\left(\mathrm{p}^{\mathrm{AA}-\mathrm{AG}}=0.01\right)$; HOMAIR, 6.1 $\pm 3.6 ; 3.4 \pm 2.0 ; 4.4 \pm 3.3 \quad\left(p^{\mathrm{AA}-\mathrm{AG}}=0.01\right)$, respectively. Leptin without statistically significant differences was elevated in AA genotype carriers $67.1 \pm 25.1$, in contrast to carriers of AG and GG genotypes: $54 \pm 26.6$; $50.8 \pm 26.5$, respectively. In girls with a gynoid obesity - carriers of AA genotype, thickness of subcutaneous adipose tissue in thighs was $2.0 \pm 0.2 \mathrm{~cm} ; A G$ genotype - 2.1 $\pm 0.3 \mathrm{~cm}$; GG-genotype $-2.5 \pm 0.8 \mathrm{~cm} \quad\left(\mathrm{p}^{\mathrm{AA}-}\right.$ $\mathrm{GG}=0.03)$. Metabolism parameters: insulin in AA genotype carriers was 13.4 \pm 5.1 ; AG genotype - 11.8 \pm 6.3 ; GG genotype $21 \pm 14.2\left(\mathrm{p}^{\mathrm{AA}-\mathrm{GG}}=0.04 ; \mathrm{p}^{\mathrm{AG}-\mathrm{GG}}=0.02\right)$. Leptin $34.5 \pm 15.7 ; 32.2$ $\pm 16.3 ; 55.8 \pm 19.7\left(\mathrm{p}^{\mathrm{AA}-\mathrm{GG}}=0.005 ; \mathrm{p}^{\mathrm{AG}-\mathrm{GG}}=0.003\right)$ respectively. HOMA-IR in carriers of AA genotype was $3.0 \pm 1.6$; AG genotype - 2.6 \pm 1.4 ; GG-genotype - $4.5 \pm 3.2\left(\mathrm{p}^{\mathrm{AG}-\mathrm{GG}}=0.04\right)$.

Conclusions In girls of android morphotype, the carriage of A-allele is associated with carbohydrate and energy metabolism disorders, and is a risk marker of excess fat deposition in chest area. For a gynoid morphotype, G-allele is a risk marker and is associated with excessive fat deposition in the thighs, as well as with carbohydrate and energy metabolism disorders.

\section{GP223 CREATINE DEFICIENCY DISORDERS THE ALL-IRELAND EXPERIENCE}

${ }^{1}$ Caoimhe Howard", 'AA Monavari, 'Ina Knerr, 'Gregory Pastores, ${ }^{2}$ Siobhan O'Sullivan, ${ }^{1}$ Ellen Crushell. ${ }^{1}$ National Centre for Inherited Metabolic Disorders, Temple Street Children's University Hospital, Dublin, Ireland; ${ }^{2}$ Department of Paediatrics, Royal Belfast Hospital for sick Children, Belfast, UK

10.1136/archdischild-2019-epa.282

Background Cerebral creatine deficiency is caused by rare inherited disorders of either creatine biosynthesis (i.e. guanidinoacetate methyltransferase (GAMT) and arginine:glycine amidinotransferase (AGAT) deficiency), or creatine transport (i.e. creatine transporter deficiency, CRTR). Clinical features include variable hypotonia, speech delay (often the dominant feature), seizures, extrapyramidal signs and behavioural issues. Patients may have feeding issues and low weight. There is some evidence that early treatment of these disorders is successful, particularly in GAMT and AGAT deficiency.

Method A retrospective chart review was undertaken of all patients who have attended the national metabolic referral centres at Dublin and Belfast with creatine deficiency. Details recorded included history, clinical findings, treatments and results of laboratory, genetic and radiological investigations.

Results Six patients with current ages between 5 and 29 years were identified with a confirmed disorder of creatine metabolism - four male patients with X-linked CRTR and two female siblings with autosomal recessive GAMT deficiency. Initial diagnosis was made on urine biochemistry (analysis of creatine/creatinine and guanidinoacetate) in two patients, on MR spectroscopy in three patients (creatine peak significantly reduced) and via exome sequencing in one patient. All cases were confirmed biochemically and genetically. Despite onset of symptoms before the age of 2 years, mean age at diagnosis was 7.5 years, with range from 20 months to 17 years.
Six patients had significant developmental delay, particularly in the domains of speech and behaviour. Three of the patients have weights $<9$ th centile. Five of the patients had documented head circumferences, and all were normocephalic proportional to height and weight. Both patients with GAMT deficiency had epilepsy which responded to treatment with creatine and ornithine. Three patients with CRTR also had epilepsy. Two patients with CRTR have been treated with creatine and creatine/arginine/glycine/S-adenosylmethionine in combination, without notable effect on clinical symptoms or MR spectroscopy findings, which is in keeping with expectations for this condition.

The four patients with CRTR are hemizygous for pathogenic mutations in the SLC6A8 gene, de novo in two patients and maternally inherited in another, one has not had parental testing. The siblings with GAMT deficiency are compound heterozygous for mutations in the GAMT gene.

Conclusion Although rare in Ireland, these treatable disorders are likely under-diagnosed. In a patient with developmental delay (particularly speech impairment) and behavioural difficulties, consideration should be given to sending a urine sample for analysis of creatine/creatinine ratio and guanidinoacetate, particularly if there is comorbid epilepsy.

\section{GP224 MULTISYSTEM MITOCHONDRIAL DISEASES IN CHILDREN WITH MATERNALLY INHERITED COMPLEX I DEFICIENCY}

Tereza Danhelovska, Hana Kolarova, Jan Langer, Kamila Berankova, Hana Hansikova, Marketa Tesarova, Tomas Honzik, Jiri Zeman*. Department of Paediatrics, First Faculty of Medicine, Charles University and General University Hospital, Prague, Czech Republic

\subsection{6/archdischild-2019-epa.283}

Mitochondrial disorders (MD) in childhood represent a heterogeneous group of disease. The most common cause of MD is respiratory chain complex I (CI) deficiency, which may be caused by mutations in either nuclear or the mitochondrial DNA (mtDNA). In the cohort of 106 unrelated families with mtDNA mutations from our region with 10,5 million of inhabitants, the multisystem MD due mtDNA mutations in MT-ND genes for structural subunits of CI were recognized in 12 families with 13 affected children.

Results In the group of 13 patients, altogether 8 different heteroplasmic mtDNA mutations in MT-ND genes were found. Mutations in MT-ND5 gene were most frequent including one novel mutation m.13091T $>$ C. Six children with the mutation heteroplasmy $>60 \%$ had Leigh syndrome and significantly worse prognosis than five patients with heteroplasmy $<60 \%$, who developed MELAS syndrome with stroke-like episodes. In last two children, the diseases started with optic neuropathy but both children transitioned later to multisystem diseases compatible with MELAS syndrome. The activities of CI in isolated muscle mitochondria were decreased in most patients and analyses with $\left[1-{ }^{14} \mathrm{C}\right]$ pyruvate, $\left[\mathrm{U}-{ }^{14} \mathrm{C}\right]$ malate and $\left[1,4-{ }^{14} \mathrm{C}\right]$ succinate substrates revealed decreased $\mathrm{CO}_{2}$ production in some patients.

Conclusions Children with the multisystem MD due to $\mathrm{CI}$ deficiency and heteroplasmic mtDNA mutations usually develop Leigh or MELAS syndromes and represent approximately $11 \%$ of families with maternally inherited MD diagnosed in our region. Early onset of the disease and higher level of heteroplasmy of mtDNA mutations resulted in Leigh 